\title{
Correspondence
}

\section{Tumor Exosome-Mediated MDSC Activation}

\section{To the Editor-in-Chief:}

In a recent issue of The American Journal of Pathology, Xiang et $\mathrm{al}^{1}$ published their results concerning the effects of tumor-derived exosomes on myeloid-derived suppressor cell (MDSC) biology. They compared the biological effects of exosomes derived from in vitro cultured B16 tumor cells (termed $\mathrm{C}$-exo for culture exosome) and exosomes derived from in vivo grown B16 tumor (termed P-exo for primary exosomes). They reported that P-exo induce Toll-like receptor 2 (TLR2)-independent MDSC activation and expansion, whereas C-exo activate and expand MDSC in a TLR2-dependent manner. These data are in contrast with our own recent article demonstrating MDSC activation through TLR2 ligation by heat shock protein 72 (Hsp72) expressed on exosomes from CT26 tumors. ${ }^{2}$ In their discussion, Xiang et al ${ }^{1}$ propose a hypothesis to explain this discrepancy between $\mathrm{C}$-exo and P-exo. Here, we provide our point of view about this difference.

Xiang et al ${ }^{1}$ propose that P-exo harbor a TLR2-independent mechanism of MDSC activation that is different from C-exo. This is probably true. Indeed, in another study from the same group, Xiang et $\mathrm{al}^{3}$ provided evidence that TS/A tumor cell lines produce exosomes containing prostaglandin $E_{2}\left(P E_{2}\right)$, and it has previously been shown that $\mathrm{PGE}_{2}$ alone is sufficient to induce MDSC expansion and activation in a TLR2-independent manner. ${ }^{4}$ However, in the models used in our study, we provide evidence that there is no detectable $\mathrm{PGE}_{2}$ in tumor-derived exosomes. Xiang et al ${ }^{1}$ assert that our work demonstrated that "tumor exosomes trigger MDSC expansion via activation of STAT3." However, this assumption is false. In our model, we demonstrated that there are indeed two distinct signals: tumor-derived exosomes account for MDSC activation (STAT3 phosphorylation and interleukin 6 secretion), whereas tumor-derived soluble factors [namely, granulocyte-macrophage colony-stimulating factor (GMCSF)] are responsible for expansion. In the EL4 model, we observed that tumors growing in $\mathrm{TLR}^{-1-}$ mice induced MDSC expansion but not MDSC activation compared with wild-type mice. Thus, we can exclude the in vitro effect hypothesized by Xiang et al, ${ }^{1}$ because, in this in vivo setting, we observed proliferation dissociated from activation. In the model by Xiang et al, for unknown reasons, C-exo induced both STAT3 activation and expansion of MDSC; however, we never observed proliferation of MDSC cultured with exosomes alone. Moreover, Xiang et al already reported that the expansion and proliferation of MDSC in their model may be related to the presence of $\mathrm{PGE}_{2}$. Indeed, we also observed that $\mathrm{PGE}_{2}$ alone could trigger MDSC expansion and proliferation, so we could postulate that exosomes may have contained $\mathrm{PGE}_{2}$ in the model of Xiang et al, whereas we could not detect $\mathrm{PGE}_{2}$ in exosomes from our own models. These data may explain the discrepancy between our works.

Furthermore, the preparation of the P-exo raises some methodological concerns. They are prepared from isolated in vivo grown tumors, with less than 5 in vitro passages. Many nontumoral cells, such as myeloid cells or fibroblasts, might be present in the preparation. These cell contaminants could be responsible for the production of $\mathrm{PGE}_{2}$-containing exosomes because it is well known that tumor-infiltrating macrophages may produce $\mathrm{PGE}_{2}{ }^{5}$ and exosomes. ${ }^{6}$

Xiang et $\mathrm{al}^{1}$ also propose that the difference between $\mathrm{C}$-exo and P-exo is based on the passage number of in vitro cultured cells. We work with cell lines obtained from the American Type Culture Collection and used at passage numbers less than 15 to 20, so we assume the derivation from the parental cell line is minimal. Xiang et al also proposed that mycoplasma contamination could be responsible for the TLR2-dependent activation signal, which would be eliminated by the in vivo passage of the tumor. In our study, we routinely tested for mycoplasma contamination, but Xiang et al suspect that the available tests are insufficient. However, in our study, we demonstrated that exosomes derived from mycoplasma-free Hsp72-silenced CT26 were unable to activate MDSC, whereas mycoplasma-free mock CT26-derived exosomes activated MDSC. Further, in vivo Hsp72-silenced tumors were unable to induce STAT3 activation of MDSC, thus supporting the premise that the Hsp72-TLR2-MyD88 pathway is vital in our model. In our opinion, this clearly excludes concerns about a potential effect of mycoplasma contamination because the Hsp72 status alone would not be a determinant otherwise.

In summary, we propose that the discrepancy identified by Xiang et $\mathrm{al}^{1}$ between $\mathrm{C}$-exo and P-exo may 
actually rely on the presence of tumor exosome-associated $\mathrm{PGE}_{2}$ produced by tumor or contaminant cells, which bypass the Hsp72-TLR2-MyD88 signal.

\section{Gregoire Mignot Fanny Chalmin Sylvain Ladoire Cédric Rébé François Ghiringhelli} INSERM U866

Dijon, France

\section{Author's Reply:}

In comparing the data published by our group ${ }^{1}$ with that published by Chalmin et al, ${ }^{2}$ the major discrepancies are that i) they did not detect $\mathrm{PGE}_{2}$ on the tumor exosomes purified from the same type of tumor cells, whereas we could detect $P G E_{2}$, and ii) our data do not support a major role for TLR2 in tumor exosomes in mediating either activation or expansion of MDSCs.

Regarding the first point, we still believe that our data closely represent what occurs in vivo because tumor exosomes play a role in the induction of chronic inflammation, ${ }^{3,7-15}$ and $\mathrm{PGE}_{2}$ released from different types of cells, including tumor cells, has the capacity to bind tumor exosomes. Therefore, $\mathrm{PGE}_{2}$ does not have to enter exosomes before they are released from the cell because association could occur after $\mathrm{PGE}_{2}$ is released from many types of cells. In the tumor microenvironment, large numbers of nontumor and tumor cells release $\mathrm{PGE}_{2}$, which subsequently binds to exosomes. This is not contamination as suggested by Ghiringhelli and colleagues. In fact, our published data of exosomes released from ex vivo cultured tumor cells ${ }^{1}$ closely represent what occurs in the tumor microenvironment. Whether $\mathrm{PGE}_{2}$ is released from tumor cells or nontumor cells should be further tested. However, the key point for designing future therapeutic strategies is that our data provide valuable information as a close reflection of what takes place in vivo.

Second, when hypothesizing that tumor exosomes play a role in immune suppression through the expansion of MDSCs, we believe it is more convincing that tumor cells have contact with immune cells, including $T$ cells, as demonstrated in our report. ${ }^{1}$ It is conceivable that exosomes released from in vitro cultured tumor cells do not have to actively sort the molecules into exosomes for immune suppression because the immune suppression is initiated after long-term in vitro culture. In contrast, the exosomes released from tumor tissue or after ex vivo culture with immune cells are expected to exhibit more potent immunosuppressive function because tumor cells possess multiple immune escape strategies. Therefore, we believe that after tumor cells communicate with immune cells, including infiltrated $T$ cells, these tumor cells develop immune escape mechanisms to avoid the host TLR2-mediated innate immune response. Whether tumor exosomes or GM-CSF alone is sufficient to cause the expansion of MDSCs is difficult to conclude, because in our systems ${ }^{1}$ bone marrow precursors cells were cultured in the presence of GM-CSF and tumor exosomes.

Finally, regardless of the strategies to eliminate the possibility of mycoplasm contamination, one should not exclude the possibility of unidentified species of mycoplasma contamination because TLR-mediated signaling is easily stimulated even with low levels of an appropriate ligand. Collectively, our data support the notion that tumor cells should be reisolated frequently from an immune-competent host for examining tumor exosome-mediated immunosuppression. Otherwise, the data generated from in vitro cultured cells are less meaningful for clinical translational research.

University of Louisville

Louisville, Kentucky

Xiaoyu Xiang
Douglas D. Taylor
William Grizzle
Huang-Ge Zhang

\section{References}

1. Xiang X, Liu Y, Zhuang X, Zhang S, Michalek S, Taylor DD, Grizzle W, Zhang HG: TLR2-mediated expansion of MDSCs is dependent on the source of tumor exosomes. Am J Pathol 2010, 177:16061610

2. Chalmin F, Ladoire S, Mignot G, Vincent J, Bruchard M, RemyMartin JP, Boireau W, Rouleau A, Simon B, Lanneau D, De Thonel A, Multhoff G, Hamman A, Martin F, Chauffert B, Solary E, Zitvogel L, Garrido C, Ryffel B, Borg C, Apetoh L, Rebe C, Ghiringhelli F: Membrane-associated Hsp72 from tumor-derived exosomes mediates STAT3-dependent immunosuppressive function of mouse and human myeloid-derived suppressor cells. J Clin Invest 2010, 120:457-471

3. Xiang X, Poliakov A, Liu C, Liu Y, Deng ZB, Wang J, Cheng Z, Shah SV, Wang GJ, Zhang L, Grizzle WE, Mobley J, Zhang HG: Induction of myeloid-derived suppressor cells by tumor exosomes. Int J Cancer 2009, 124:2621-2633

4. Sinha P, Clements VK, Fulton AM, Ostrand-Rosenberg S: Prostaglandin E2 promotes tumor progression by inducing myeloid-derived suppressor cells. Cancer Res 2007, 67:4507-4513

5. Malick AP, Elgert KD, Garner RE, Adkinson NF Jr: Prostaglandin E2 production by Mac-2+ macrophages: tumor-induced population shift. J Leukoc Biol 1987, 42:673-681

6. Thery C, Ostrowski M, Segura E: Membrane vesicles as conveyors of immune responses. Nat Rev Immunol 2009, 9:581-593

7. Liu Y, Xiang X, Zhuang X, Zhang S, Liu C, Cheng Z, Michalek S, Grizzle W, Zhang HG: Contribution of MyD88 to the tumor exosomemediated induction of myeloid derived suppressor cells. Am J Pathol 176:2490-2499

8. Qu JL, Qu XJ, Zhao MF, Teng YE, Zhang Y, Hou KZ, Jiang YH, Yang $\mathrm{XH}$, Liu YP: Gastric cancer exosomes promote tumour cell proliferation through PI3K/Akt and MAPK/ERK activation. Dig Liver Dis 2009, 41:875-880

9. Huber $V$, Filipazzi $P$, lero M, Fais S, Rivoltini L: More insights into the immunosuppressive potential of tumor exosomes. J Transl Med 2008 6:63 
10. Ichim TE, Zhong Z, Kaushal S, Zheng X, Ren X, Hao X, Joyce JA, Hanley HH, Riordan NH, Koropatnick J, Bogin V, Minev BR, Min WP. Tullis $\mathrm{RH}$ : Exosomes as a tumor immune escape mechanism: possible therapeutic implications. J TransI Med 2008, 6:37

11. Clayton A, Mitchell JP, Court J, Mason MD, Tabi Z: Human tumorderived exosomes selectively impair lymphocyte responses to interleukin-2. Cancer Res 2007, 67:7458-7466

12. Yu S, Liu C, Su K, Wang J, Liu Y, Zhang L, Li C, Cong Y, Kimberly R, Grizzle WE, Falkson C, Zhang HG: Tumor exosomes inhibit differentiation of bone marrow dendritic cells. J Immunol 2007, 178:68676875
13. Valenti R, Huber V, Iero M, Filipazzi P, Parmiani G, Rivoltini L: Tumorreleased microvesicles as vehicles of immunosuppression. Cancer Res 2007, 67:2912-2915

14. Wieckowski E, Whiteside TL: Human tumor-derived vs dendritic cellderived exosomes have distinct biologic roles and molecular profiles. Immunol Res 2006, 36:247-254

15. Valenti R, Huber V, Filipazzi P, Pilla L, Sovena G, Villa A, Corbelli A, Fais S, Parmiani G, Rivoltini L: Human tumor-released microvesicles promote the differentiation of myeloid cells with transforming growth factor-beta-mediated suppressive activity on T Iymphocytes. Cancer Res 2006, 66:9290-9298 